\title{
APEX telescope observations of new molecular ions
}

\author{
F. Wyrowski, K. M. Menten, R. Güsten and A. Belloche \\ Max-Planck-Institut für Radioastronomie, Auf dem Hügel 69, 53121 Bonn, Germany
}

\begin{abstract}
Hydrides are key ingredients of interstellar chemistry since they are the initial products of chemical networks that lead to the formation of more complex molecules. The fundamental rotational transitions of light hydrides fall into the submillimeter wavelength range. Using the APEX telescope, we observed the long sought hydrides $\mathrm{SH}^{+}$and $\mathrm{OH}^{+}$in absorption against the strong continuum source Sagittarius B2(M). Both, absorption from Galactic center gas as well as from diffuse clouds in intervening spiral arms over a large velocity range is observed. The detected absorption of a continuous velocity range on the line of sight shows these hydrides to be an abundant component of diffuse clouds. In addition, we used the strongest submillimeter dust continuum sources in the inner Galaxy to serve as background candles for a systematic census of these hydrides in diffuse clouds and massive star forming regions of our Galaxy and initial results of this survey are presented.
\end{abstract}

Keywords: Astrochemistry — ISM: abundances — ISM - molecules

PACS: <Replace this text with PACS numbers; choose from this list: http://www.aip. .org/pacs/index.html>

\section{INTERSTELLAR HYDRIDES}

Hydrides were the first molecules detected in interstellar space. More then 70 years ago, optical absorption lines from $\mathrm{CH}$ and $\mathrm{CH}^{+}$were detected in stellar spectra by Dunham [1] due to intervening interstellar diffuse clouds on the lines-of-sight. Diffuse clouds are relatively tenuous, exposed to the UV radiation from stars. A comprehensive review of diffuse clouds and the molecular detections in them is given in Snow and McCall [2]. Hydrides are key ingredients of interstellar chemistry since they are the initial products of chemical networks that lead to the formation of more complex molecules. The fundamental rotational transitions of light hydrides fall into the submillimeter wavelength range, among them also several transitions of the water molecule. As an abundant component of the earth's atmosphere, water is responsible for blocking large portion of the submillimeter wavelengths range for ground-based observations. Only from exceptional sites, such as the the dry high altitude Chajnantor plateau in the Atacama desert of northern Chile, reasonable transparency in several atmospheric windows is reached over substantial time periods that is exploited by the APEX, ASTE and NANTEN2 single dish telescopes and soon the Atacama Large Millimeter Array interferometer (ALMA). For a review of hydride discoveries using the Caltech Submillimeter Observatory (CSO) on Mauna Kea see Lis et al. [3]. Here we summarize recent new observations of interstellar hydrides using the Atacama Pathfinder EXperiment, APEX, see Güsten et al. [4], that complement the flood of new hydride results obtained with the Herschel Space Observatory.

\section{NEW MOLECULAR IONS TOWARDS SGR B2 (M)}

Sagittarius B2 is the most massive star forming region in our Galaxy. It harbors the most luminous hot molecular cores, Sgr B2(M) and (N), and is situated close to the Galactic Center at a distance of about $8 \mathrm{kpc}$. The high gas column density, high excitation and rich chemistry make this region the best hunting ground for new interstellar molecules and many of the molecules were indeed firstly discovered there. In addition, the line of sight towards the source crosses several galactic spiral arms, which enables absorption studies of intervening diffuse clouds. The strong continuum emission of Sgr B2 is due to luminous ultracompact HII regions at centimeter wavelengths and thermal dust continuum emission from their massive envelopes in the submillimeter/far-infrared.

The 12m APEX telescope, a modified copy of the US ALMA prototype antenna, was used in conjunction with the powerful CHAMP+ heterodyne array described in Kasemann et al. [7] to observe toward Sgr B2(M) several hydrides in the 450 and $350 \mu \mathrm{m}$ atmospheric windows, two of them for the first time in interstellar space. Figure 1 shows the measured spectra of ${ }^{13} \mathrm{CH}^{+}, \mathrm{HCl}, \mathrm{OH}^{+}$and $\mathrm{SH}^{+}$together with observations of $\mathrm{H}^{13} \mathrm{CO}^{+}$and $\mathrm{C}_{3} \mathrm{H}_{2}$ taken from a $3 \mathrm{~mm}$ line survey carried out with the IRAM 30m telescope for comparison (see Belloche et al. [8]). Besides strong 


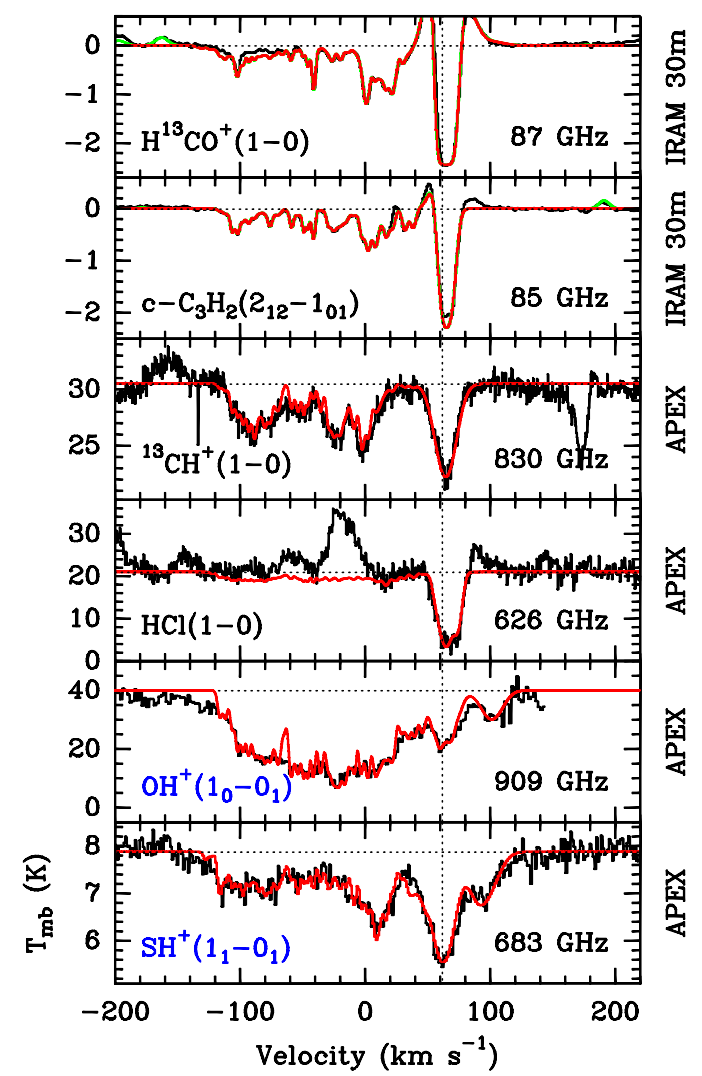

FIGURE 1. New hydrides observations with APEX towards Sgr B2(M) (Menten et al. [5], Wyrowski et al. [6]; the upper two panels show IRAM 30m detections for comparison).

absorption at the source velocity around $65 \mathrm{~km} \mathrm{~s}^{-1}$, all molecules except $\mathrm{HCl}$ show in addition absorption from a large range of velocities from diffuse clouds on the line of sight. This demonstrates that the newly detected molecular ions $\mathrm{SH}^{+}$and $\mathrm{OH}^{+}$are an abundant component of diffuse clouds (Menten et al. [5], Wyrowski et al. [6]).

At the velocity of the Sgr B2(M) cloud, the $\mathrm{SH}^{+}$column density is $2 \times 10^{14} \mathrm{~cm}^{-2}$ and in the diffuse line-ofsight clouds column densities per unit velocity interval of 0.5 to $6 \times 10^{12} \mathrm{~cm}^{-2} /\left(\mathrm{km} \mathrm{s}^{-1}\right)$ are found. ${ }^{13} \mathrm{CH}^{+}$can be used together with the Galactic gradient of the ${ }^{12} \mathrm{C} /{ }^{13} \mathrm{C}$ ratio measured by Milam et al. [9] to determine the column densities of $\mathrm{CH}^{+}$. The $\mathrm{SH}^{+} /{ }^{12} \mathrm{CH}^{+}$column density ratio varies significantly between velocity components, from 0.07 to 1.2 .

An $\mathrm{OH}^{+}$column density local to Sgr B2(M) of $2.6 \times 10^{14} \mathrm{~cm}^{-2}$ is found. On the intervening line of sight, the column density per unit velocity interval is in the range of 1 to $40 \times 10^{12} \mathrm{~cm}^{-2} /\left(\mathrm{km} \mathrm{s}^{-1}\right)$. $\mathrm{OH}^{+}$is found to be on average more abundant than the other hydrides $\mathrm{SH}^{+}$and $\mathrm{CH}^{+}$. Abundance ratios of $\mathrm{OH}$ and atomic oxygen to $\mathrm{OH}^{+}$ are found in the range of $10^{1-2}$ and $10^{3-4}$, respectively.

\section{INNER GALAXY SURVEY OF OH ${ }^{+}$}

The rotational ground state transition of $\mathrm{OH}^{+}$is split into three lines at 909,971 and $1033 \mathrm{GHz}$, each of them showing further hyperfine splitting. Besides the $909 \mathrm{GHz}$ lines, also the $1033 \mathrm{GHz}$ line can be observed from the ground from an excellent site such as the Chajnantor plateau in the first atmospheric $\mathrm{THz}$ window. This was done for the first time in June 2010 with the APEX telescope using the new MPIfR THz receiver (Wyrowski et al., Leinz et al., both in prep.). Since the $1033 \mathrm{GHz}$ lines are stronger than the $909 \mathrm{GHz}$ lines, they allow conducting a survey of $\mathrm{OH}^{+}$towards strong continuum sources in a reasonable amount of time. Twenty-five of the strongest submillimeter continuum sources, all luminous massive star forming regions, were observed in the inner Galaxy during excellent atmospheric conditions with precipitable water vapor of about $0.2-0.3 \mathrm{~mm}$, resulting in atmospheric transmission of up to $30 \%$. $\mathrm{OH}^{+}$could be 


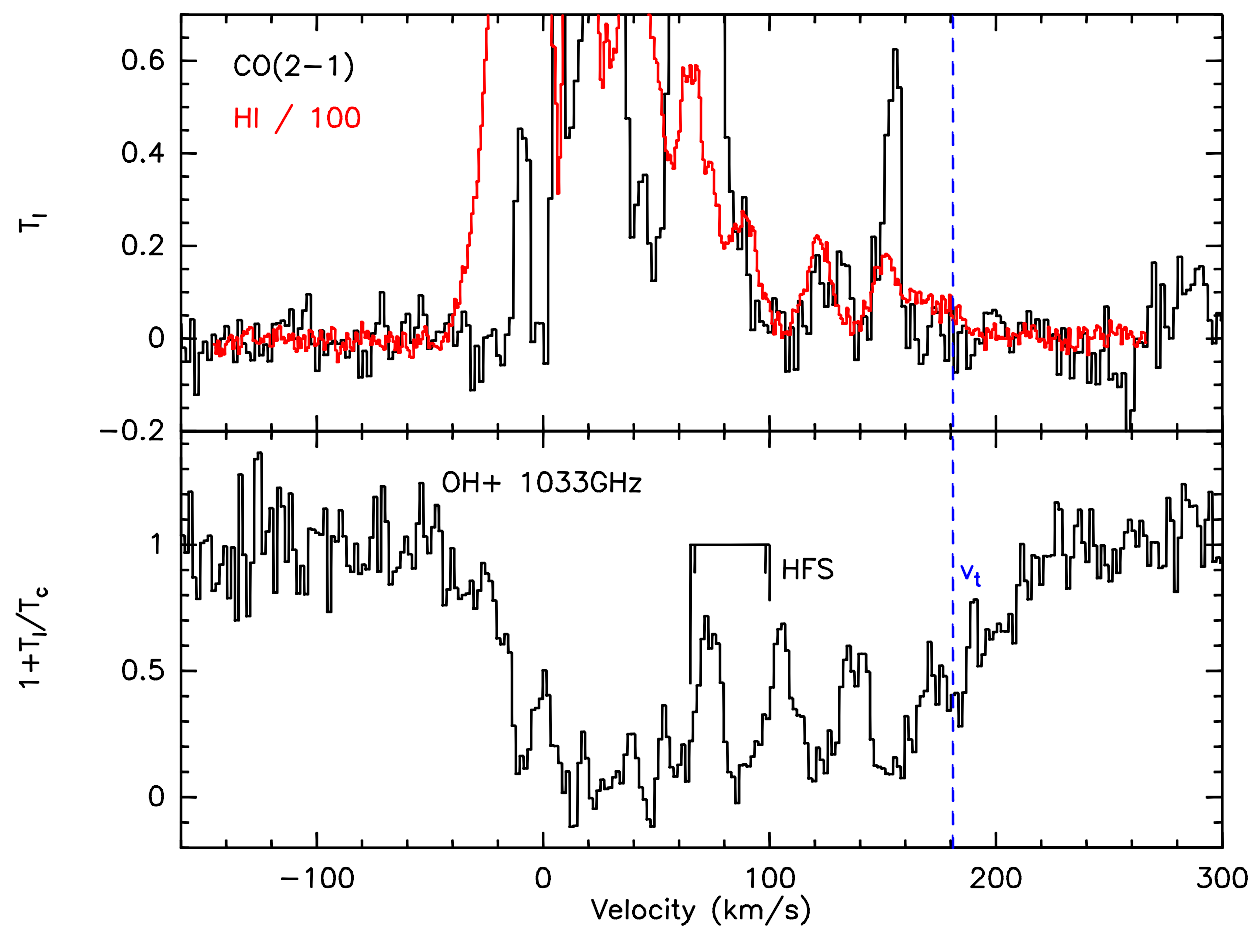

FIGURE 2. APEX THz observations of $\mathrm{OH}^{+}$towards the massive star forming clump G10.5+0.0 (lower panel), compared with $\mathrm{CO}(2-1)$ (APEX) and HI (divided by 100, extracted from the Southern Galactic Plane Survey, McClure-Griffiths et al. [10]). The hyperfine structure pattern of $\mathrm{OH}^{+}$as well as the the tangential velocity at the source galactic longitude are indicated.

detected towards most of the sources as a further confirmation for the widespread distribution of $\mathrm{OH}^{+}$in the interstellar medium. Figure 2 shows as a preliminary result the deep absorption found towards the massive star forming clump G10.47+0.03, which is much broader than the maximal separation of hyperfine components of about $35 \mathrm{~km} \mathrm{~s}^{-1}$. Comparing the absorption with emission of $\mathrm{CO}$ and $\mathrm{HI}$ towards this source, it is found that all of the $\mathrm{OH}^{+}$absorption components have $\mathrm{HI}$ counterparts, while in a few $\mathrm{OH}^{+}$velocity ranges, no $\mathrm{CO}$ was detected. This is especially evident at the extreme velocities close to the tangential velocity on this line of sight. Since the velocity of the star forming region itself is at about $60 \mathrm{~km} \mathrm{~s}^{-1}$, the detection of velocity components beyond the source velocity shows that the region is situated at the far kinematic distance at a distance of $10.6 \mathrm{kpc}$, which is consistent with the HI absorption results by Pandian et al. [11]. This result shows that $\mathrm{OH}^{+}$absorption studies might be employed to break the near/far kinematic distance ambiguity for sources that show only strong submillimeter continuum and no centimeter continuum needed for HI absorption studies.

\section{PROSPECTS FOR ALMA}

The ground-based hydride observations with the CSO (Lis et al. [3]) and APEX pave the way for future studies with high angular resolution and sensitivity using the ALMA interferometer, currently being constructed on the Chajnantor plateau, with early science likely starting already late in 2011. Table 1 summarizes important hydride lines that will be observable with ALMA. For sources with considerable redshift even more hydride transitions come into reach, e.g. ground state lines from $\mathrm{CH}^{+}, \mathrm{H}_{2} \mathrm{O}, \mathrm{H}_{3} \mathrm{O}^{+}, \mathrm{HF}$ and $\mathrm{NH}^{+}$, although the latter has so far not been detected in interstellar space. The recent Herschel extragalactic detections of $\mathrm{H}_{2} \mathrm{O}^{+}$in nearby starburst galaxies (Weiss et al. [12], Van der Werf et al. [13]) offer promising prospects for high redshift detections. The usual excellent bandpass stability of interferometers will facilitate those detections. ALMA will be able to map the absorption in front of the spatially extended submillimeter dust continuum. This will allow to study the small scale structure of the hydrides in the diffuse clouds in conjunction with high velocity resolution of the lines. ALMA will also allow detailed mapping of hydrides 
in the outflows from young stellar objects to shed light onto the detailed chemical processes in the flows.

TABLE 1. Rotational lines of hydrides accessible with ALMA from the ground. In case of hyperfine structure, only the strongest component is given. References to astronomical observations of the lines are given.

\begin{tabular}{lclrl}
\hline Molecule & Transition & $\begin{array}{l}\text { Frequency } \\
(\mathrm{GHz})\end{array}$ & Band & Reference/Note \\
\hline $\mathrm{OH}^{+}$ & $1-0$ & 909.1588 & 10 & Wyrowski et al. [6] \\
$\mathrm{SH}^{+}$ & $1-0$ & 683.4223 & 9 & Menten et al. [5] \\
$\mathrm{SH}^{+}$ & $1-0$ & 345.9298 & 7 & close to CO \\
$\mathrm{SH}^{+}$ & $2-1$ & 893.1338 & 10 & Menten et al. [5] \\
${ }^{13} \mathrm{CH}^{+}$ & $1-0$ & 830.2161 & 10 & Menten et al. [5] \\
$\mathrm{HCl}^{+}$ & $1-0$ & 625.9188 & 9 & Menten et al. [5] \\
$\mathrm{H}_{2} \mathrm{Cl}^{+}$ & $1-0$ & 485.4208 & 8 & Lis et al. [14] \\
$\mathrm{H}_{2} \mathrm{O}^{+}$ & $3 / 2-1 / 2$ & 604.6786 & 9 & Schilke et al. [15] \\
$\mathrm{H}_{2} \mathrm{O}^{+}$ & $3 / 2-3 / 2$ & 607.2273 & 9 & Schilke et al. [15] \\
$\mathrm{H}_{2} \mathrm{O}^{+}$ & $1 / 2-1 / 2$ & 631.7241 & 9 & Schilke et al. [15] \\
$\mathrm{H}_{2} \mathrm{O}^{+}$ & $1 / 2-3 / 2$ & 634.2729 & 9 & Schilke et al. [15] \\
$\mathrm{NH}$ & $1-0$ & 946.4758 & 10 & Hily-Blant et al. [16] \\
$\mathrm{NH}_{2}$ & $3 / 2-3 / 2$ & 462.4335 & 8 & van Dishoeck et al. [17] \\
\hline
\end{tabular}

\section{REFERENCES}

1. T. Dunham, PASP 49, 26-28 (1937).

2. T. P. Snow, and B. J. McCall, ARA\&A 44, 367-414 (2006).

3. D. C. Lis, P. F. Goldsmith, E. A. Bergin, E. Falgarone, M. Gerin, and E. Roueff, "Hydrides in Space: Past, Present, and Future," in Astronomical Society of the Pacific Conference Series, edited by D. C. Lis, J. E. Vaillancourt, P. F. Goldsmith, T. A. Bell, N. Z. Scoville, \& J. Zmuidzinas, 2009, vol. 417 of Astronomical Society of the Pacific Conference Series, pp. 23-24.

4. R. Güsten, L. Å. Nyman, P. Schilke, K. Menten, C. Cesarsky, and R. Booth, A\&A 454, L13-L16 (2006).

5. K. Menten, F. Wyrowski, A. Belloche, R. Güsten, H. Müller, and L. Dedes, A\&A in press, preprint arXiv:1009.2825 (2010).

6. F. Wyrowski, K. M. Menten, R. Güsten, and A. Belloche, A\&A 518, A26+ (2010).

7. C. Kasemann, R. Güsten, S. Heyminck, B. Klein, T. Klein, S. D. Philipp, A. Korn, G. Schneider, A. Henseler, A. Baryshev, and T. M. Klapwijk, "CHAMP+: a powerful array receiver for APEX," in Society of Photo-Optical Instrumentation Engineers (SPIE) Conference Series, 2006, vol. 6275 of Society of Photo-Optical Instrumentation Engineers (SPIE) Conference Series.

8. A. Belloche, K. M. Menten, C. Comito, H. S. P. Müller, P. Schilke, J. Ott, S. Thorwirth, and C. Hieret, A\&A 482, 179-196 (2008), 0801.3219.

9. S. N. Milam, C. Savage, M. A. Brewster, L. M. Ziurys, and S. Wyckoff, ApJ 634, 1126-1132 (2005).

10. N. M. McClure-Griffiths, J. Dickey, B. M. Gaensler, A. J. Green, M. Haverkorn, and S. S., ApJS 158, $178-187$ (2005).

11. J. Pandian, E. Momjian, and P. Goldsmith, A\&A 486, 191-208 (2008).

12. Weiss et al., $A \& A$ in press (2010).

13. Van der Werf et al., A\&A 518, L42 (2010).

14. D. C. Lis, J. C. Pearson, and D. A. Neufeld et al., A\&A in press, preprint arXiv:1007.1461 (2010).

15. P. Schilke, C. Comito, and H. S. P. Müller et al., A\&A in press, preprint arXiv:1007:0670 (2010).

16. Hily-Blant et al., $A \& A$ in press (2010).

17. E. F. van Dishoeck, D. J. Jansen, P. Schilke, and T. G. Phillips, ApJ 416, L83-86 (1993). 